\title{
Physical Modeling of the Zodiacal Dust Cloud
}

\author{
Leonid M. Ozernoy \\ 5C3, School of Computational Sciences and Department of Physics $\mathcal{G}$ \\ Astronomy, George Mason U., Fairfax, VA 22030-4444, USA
}

\begin{abstract}
.
This review is based on extensive work done in collaboration with N. Gorkavyi, J. Mather, and T. Taidakova, which aimed at physical modeling of the interplanetary dust (IPD) cloud in the Solar System, i.e., establishing a link between the observable characteristics of the zodiacal cloud and the dynamical and physical properties of the parent minor bodies. Our computational approach permits one to integrate the trajectories of hundreds of particles and to effectively store up to $10^{10-11}$ positions with modest computer resources, providing a high fidelity 3D distribution of the dust. Our numerical codes account for the major dynamical effects that govern the motion of IPD particles: Poynting-Robertson (P-R) drag and solar wind drag; solar radiation pressure; particle evaporation; gravitational scattering by the planets; and the influence of mean-motion resonances. The incorporation of secular resonances and collisions of dust particles (both mutual and with interstellar dust) is underway. We have demonstrated the efficacy of our codes by performing the following analyses: (i) simulation of the distribution of Centaurs (comets scattered in their journey from the Kuiper belt inward in the Solar System) and revealing the effects of the outer planets in producing 'cometary belts'; (ii) detailed inspection of a rich resonant structure found in these belts, which predicts the existence of gaps similar to the Kirkwood gaps in the main asteroid belt; (iii) a preliminary 3-D physical model of the IPD cloud, which includes three dust components - asteroidal, cometary, and kuiperoidal - and is consistent with the available data of Pioneer and Voyager dust detectors; (iv) modeling of the IPD cloud, which provides a zodiacal light distribution in accord, to the order of $1 \%$, with a subset of the COBE/DIRBE observations; and (v) showing that the resonant structure in dusty circumstellar disks of Vega and Epsilon Eridani is a signature of embedded extrasolar planets. Further improvements of our modeling and their importance for astronomy and cosmology are outlined.
\end{abstract}

\section{Introduction}

The necessity to account accurately for zodiacal emission as part of the measurements of the cosmic infrared background has revived and strengthened a long-standing interest in the theoretical aspects of the dynamics, structure, and evolution of the interpanetary dust (IPD) cloud (e.g., Hauser et al. 1998). 
Until recently, the main stumbling block to implementing comprehensive study of the IPD has been the absence of a physical model for the interplanetary dust cloud. Such a model would establish a link between the observable characteristics of the zodiacal cloud and the dynamical and physical properties of the parent minor bodies of the Solar System. Recently, we constructed a preliminary physical model of the IPD cloud based on combining new methods elaborated by the present authors (Gorkavyi, Ozernoy \& Mather [GOM hereinafter] 1996; GOM 1997; Gorkavyi et al. [GOMT hereinafter] 1997a-c; GOMT 1998a-c, 2000a-c) and some features of previously used numerical and analytical methods (Haug 1958; Kessler 1981; Leinert et al. 1983; Dermott et al. 1996; Valsecchi \& Manara 1997; Liou \& Zook 1999). Specifically, our approach, which is based on the use of the kinetic equation for the density of dust in the space of orbital coordinates as well as in ordinary space, combines analytical (kinetic or hydrodynamical) methods in conjunction with celestial mechanics orbit calculations and numerical computations. More recently, we have substantially strengthened the computational component of our approach by developing a new, more powerful technique described in the next section, which enables us to get rid of a number of approximations used before and therefore considerably improve our physical modeling.

\section{Employing A New Computational Approach}

The development of the physical model of the IPD cloud is complicated by:

- uncertainties in the distribution of comets or other bodies as the major sources of dust in planetary systems;

- difficulties in specifying accurately the level and times (positions) of dust contribution by the objects; and

- the wide variety of relevant physical processes, such as (i) radiation pressure and dissipative effects (Poynting-Robertson drag and stellar wind drag), (ii) resonant interaction with planets, (iii) gravitational scattering by planets, (iv) evaporation and sputtering of dust particles, (v) mutual collisions in the cometary and dust populations, and (vi) orbital variations in the dust production rate by minor bodies.

These complexities exclude attaining an analytical solution, so reliance must be on the numerical modeling. In our approach, two features are worth mentioning: (1) the techniques employed permit construction of high-quality 3D models of IPD clouds with the number of particles (strictly speaking, particle positions) as high as $10^{(10-11)}$; and (2) incorporation of an original stable numerical integrator suitable for both dissipationless and, with some modification, dissipational dynamics of minor bodies or particles.

\subsection{Our Computational Approach}

To date, numerical models suffered from the limited number of particles that could be used in the computations. For instance, so far the best results in modeling the dynamics of dust from the Kuiper belt were obtained by Liou \& 
Zook (1999) with $50-100$ particles of three different sizes using $\sim 10^{4}$ positions for each particle, giving $\sim 10^{6}$ particle positions. Those authors used their results for 2-D projection of the zodiacal cloud with a resolution of $1 \mathrm{AU}$ and typical statistics of 200-400 particles/ $\mathrm{AU}^{2}$ (or 20-40 particles/ $\mathrm{AU}^{3}$ for a 3-D model). Such modeling cannot easily match the large-scale structure of the IPD cloud, the respective maps of the zodiacal emission, etc.

In our approach, described in Ozernoy, Gorkavyi, \& Taidakova [OGT hereinafter] $(2000 \mathrm{a}, \mathrm{b})$ and Ozernoy, Gorkavyi, Mather \& Taidakova [OGMT hereinafter] (2000), the particle-number limitation is substantially relaxed, which is decisive to provide reliable numerical simulations. In brief, our approach is as follows (for simplicity of understanding, we consider a stationary dust particle distribution in the frame co-rotating with the planet). The locus of the given particle's positions (taken, say, as $6 \cdot 10^{3}$ positions every revolution about the star) are recorded and considered as the positions of many other particles produced by the same source of dust but at a different time. After this particle 'dies' (as a result of infall or ejection from the system by a planet-perturber), its recorded positions sampled over its lifetime form a stationary distribution as if it were produced by many particles. Typically, each run includes $10^{4}-10^{5}$ revolutions, i.e. $\sim 10^{8}$ positions of a dust particle, which is equivalent, for a stationary distribution, to $10^{8}$ particles. If we allow for 100 sources of dust (in fact, we can include, if necessary, a larger number of sources), after 100 runs we deal with $\sim 10^{10}$ particle positions as if they were real particles. In the present project, we will not only keep information about the dynamical path of each particle (as we did in OGT 2000a,b), but in addition, we will immediately sort the information about the computed coordinates of each particle into $10^{6}-10^{7}$ spatial cells (each cell containing $10^{3}-10^{4}$ particles), thereby forming a 3D grid that models the dust cloud around the Sun (or a star) (OGMT 2000). An appreciable increase in statistics, compared to Liou \& Zook (1999), brings a factor of $10^{4}$ improvement in detail of a model and enables us to model the IPD cloud at a qualitatively new, 3-D level. Moreover, our approach makes it possible to study, besides stationary processes, certain non-stationary processes as well, e.g., evolution toward steady-state distributions, dust production from non-steady sources, decrease in particle size (due to evaporation and sputtering) and number (due to collisions), etc.

\subsection{Numerical Integrators for Dissipationless and Dissipative Sys- tems}

Our computational method, as well as the use of an implicit second-order integrator (Taidakova 1997) appropriately adapted to achieve our goals (Taidakova \& Gorkavyi 1999), are described in more detail in OGT (2000b); as shown there, the integrator for a dissipationless system provides the necessary accuracy of computations on a time scale of $0.5 \cdot 10^{9}$ years. A big advantage of this integrator is its stability: an error in the energy (the Tisserand parameter) does not grow as the number of time steps increases if the value of the step remains the same. The latter situation is exemplified by a resonant particle - it does not approach too close to the planet so that the same time step can be taken. Meanwhile non-resonant particles, in due course of their gravitational scatterings, approach one or another planet from time to time, and therefore one has 
to change the time step near the planet. Obviously, whenever the time step diminishes near the planet, an error in the Tisserand parameter slowly grows together with the increased number of the smaller time steps. Nevertheless, in our simulations a fractional error in the Tisserand parameter typically does not exceed 0.001 during $3 \cdot 10^{6}$ Neptune revolutions (OGT 2000b), which amounts to 0.5 Gyrs. To increase the accuracy of the computations, we use a second iteration (OGT 2000a). While the 1st iteration yields the gravitational field between points $A$ and $B$ using an approximative formula based on the particle parameters at point $A$ (because those at point $B$ are still unknown), the 2nd iteration enables us to compute the gravitational field between $A$ and $B$ using a middle position between them because the position of $B$ is already given by the 1st iteration.

As for dissipative systems (e.g., with a P-R drag), a modified implicit second-order integrator has been elaborated (Taidakova and Gorkavyi 1999). It provides a necessary accuracy of integration, which remains stable if the time step of computations is changed in jumps, and not continuously, as the particle approaches the Sun. By applying this approach, one can compute the dynamical evolution of dust particles accounting for virtually all physical processes listed above.

\section{Components of the Interplanetary Dust Cloud}

\subsection{Asteroidal Component of Dust}

Under the bombardment of other asteroids and large grains, each asteroid serves as a source of dust. The asteroidal dust gradually approaches the Sun due to the P-R drag or escapes from the Solar System due to gravitational scattering by Jupiter and radiation pressure (for small particles) and solar wind (for small to moderate sized particles, depending on their charge, etc.).

The asteroidal component of the IPD has the following features: (i) its distribution is flat; (ii) the dust density profile is expected to be $R^{-1}$ at $R \sim$ $1 \mathrm{AU}$ and have a cut-off at $R>2$ AU (GOMT 1997a), $R$ being heliocentric distance; (iii) the asteroidal dust is mainly responsible for the content of the Earth resonant ring (Jackson \& Zook 1989; Dermott et al. 1994). Using the 'dust bands' data, Dermott et al. (1996) estimate the fraction of asteroidal particles in the IPD cloud to be about $1 / 3$.

\subsection{Cometary Component of Dust}

The cometary component of dust originates from sublimation of comets and has the following features: (i) its distribution is relatively thick; (ii) the dust density profile is expected to be $R^{-2.4}$ at $R \sim 1$ AU (GOMT 1997a); (iii) most of the cometary dust escapes from our planetary system due to perturbations by Jupiter and the solar radiation pressure. Except for a few attempts (Liou et al. 1996; GOMT 1997a), there are so far no reliable estimates for the cometary dust fraction in the IPD cloud. 


\subsection{Dust From the Kuiper Belt Objects}

The sources of the IPD cloud cannot be entirely reduced simply to comets (a part of which is also responsible for the observed dust tails) and to asteroids (a part of which assembled in asteroid families is also responsible for the observed 'dust bands' in the IPD emission) - a number of facts forces us to suspect that additional sources of interplanetary dust must exist:

1. Chemical analyses indicate that a part of IPD spends a much longer time in space than the typical asteroidal and cometary particles (Flynn 1994, 1996).

2. Pioneer 10 and 11 data indicate that the dust particles of mass $10^{-(8-9)} \mathrm{g}$ have approximately constant flux seen up to $18 \mathrm{AU}$ (Humes 1980; Divine 1993). Similarly, Voyager 1 and 2 data indicate that dust particles of mass $10^{-(11-12)} \mathrm{g}$ are seen from 6 to $40 \mathrm{AU}$ with approximately constant flux $(0.5-1) \cdot 10^{-3}$ particles $/ \mathrm{m}^{2} / \mathrm{s}$ (Gurnett et al. 1997). Neither of these results can be explained by the cometary and asteroidal sources, which give an entirely different distribution of dust (GOMT 1997a).

3. The total number of Kuiper Belt Objects (KBOs) inferred from available observations is $8 \cdot 10^{8}$ (Jewitt 1999), which exceeds the number of known Jupiter family comets by a factor of $10^{6}$. This indicates that the overall dust production rate from KBOs may not be negligible compared to that of comets, and hence a third important component of the IPD cloud might be the 'kuiperoidal' dust, as recently suspected (Backman et al. 1995).

In our opinion, the Kuiper belt influences the formation of the IPD cloud in two ways: (i) as a source of small-size particles slowly drifting toward the Sun under a combined action of the P-R drag and perturbations from the planets; and (ii) as a source of millions of comets between Jupiter and Neptune (Levison \& Duncan 1997; OGT 2000a,b), which, in turn, serve as additional sources of dust. The dust can be produced through evaporation of the volatile material from the KBO surface as a result of a variety of processes, such as the Solar wind and the heating by the Sun, micrometeor bombardment, mutual collisions of kuiperoids (e.g., Stern 2000), etc. Although these processes are very complicated, further work could enable us to put important constraints on the contribution of kuiperoidal dust in the overall dust balance.

So far, there are no reliable estimates of the kuiperoidal dust fraction in the inner Solar System. Our working hypothesis quantitatively analyzed below is that KBOs and Centaurs (invisible comets mainly beyond Jupiter) could produce an important contribution to the dust content of the IPD cloud.

\section{Simulating the Distributions of Dust Sources and Interplanetary Dust}

\subsection{The Simulated Distribution of Dust Sources in the Outer Solar System}

The outer Solar System beyond the four giant planets includes the Kuiper belt and the Oort cloud, which contain raw material remnant since the formation of the system. The KBOs are thought to be responsible for progressive replenishment of the observable cometary populations, and gravitational scattering of these objects by the four giant planets can provide their transport from the 
trans-Neptunian region all the way inward, down to Jupiter (Levison \& Duncan 1997; Malhotra, Duncan \& Levison 1999; OGT 2000a,b). An approach started in OGT 2000a,b places emphasis on the structure of cometary populations between Neptune and Jupiter, both in phase space, i.e., in the space of orbital coordinates $\{a, e, i\}$, and in real space. Using numerical simulations, we examined the structure of a cometary population near a massive planet, such as a giant planet of the Solar System, starting with a one-planet approximation (the Sun plus one planet). By studying the distributions of comets in semimajor axis, eccentricity, pericenter, and apocenter distances, we revealed several interesting features in these distributions. The most remarkable ones include: (i) each giant planet dynamically controls a cometary population that we call the 'cometary belt ${ }^{\prime}$ ); and, (ii) avoidance of resonant orbits by comets. We then enhanced the calculations by determining how a cometary belt is modified when the influence of all eight planets is taken into consideration. To this end, we simulated a stationary distribution of comets, which results from the gravitational scattering of the Kuiper belt objects mainly by the four giant planets and takes into account the effects of mean motion resonances. The objects start from the Kuiper belt and are typically traced until the bulk of comets $(\sim 90 \%)$ are ejected from the Solar System (this happens on a time scale of $\leqslant 0.5 \mathrm{Gyrs}$ ). Accounting for the influence of the four giant planets makes the simulated cometary belts overlap (Fig. 1a), but nevertheless keeps almost all of their basic features found in the one-planet approximation. In particular, the simulated belts maintain the gaps in the $(a, e)$ - and $(a, i)$-space similar to the Kirkwood gaps in the main asteroid belt.

The simulated spatial accumulations of comets near the orbits of all four giant planets - the cometary belts - have a dynamical nature, because the comets belonging to the given planet's belt are either in a resonance with the host or are gravitationally scattered predominantly by this planet. We conclude that the large-scale structure of the Solar System includes the four cometary belts expected to contain at present 20-30 million scattered comets. Only a tiny fraction of them is currently visible as Jupiter-, Saturn-, etc. family comets.

\subsection{Simulations of the Dust Distribution from Kuiper Belt Objects}

Knowledge of the simulated distribution of sources of dust, along with the known sources, has enabled us to compute the structure of the asteroidal, cometary, and kuiperoidal components of the IPD cloud. Here, we describe the structure of the latter.

In accordance with the main dynamical factors, we would expect to get three major components of the kuiperoidal dust: i) 'freely' drifting particles, (ii) gravitationally scattered particles, and (iii) particles captured into resonances.

In our phase space, we find the dust distribution highly non-uniform, with most of the dust concentrating in the four belts associated with the orbits of the four giant planets, the Neptune dust belt being the most dense and extended (Fig. 1b). As distinct from the simulated cometary belts described in Sec. 4.1, for which the dominating gravitational scattering results in avoidance of resonant orbits by comets, the dust belts, due to an additional factor - the P-R drag reveal a rich and complex resonant structure of captured particles. 

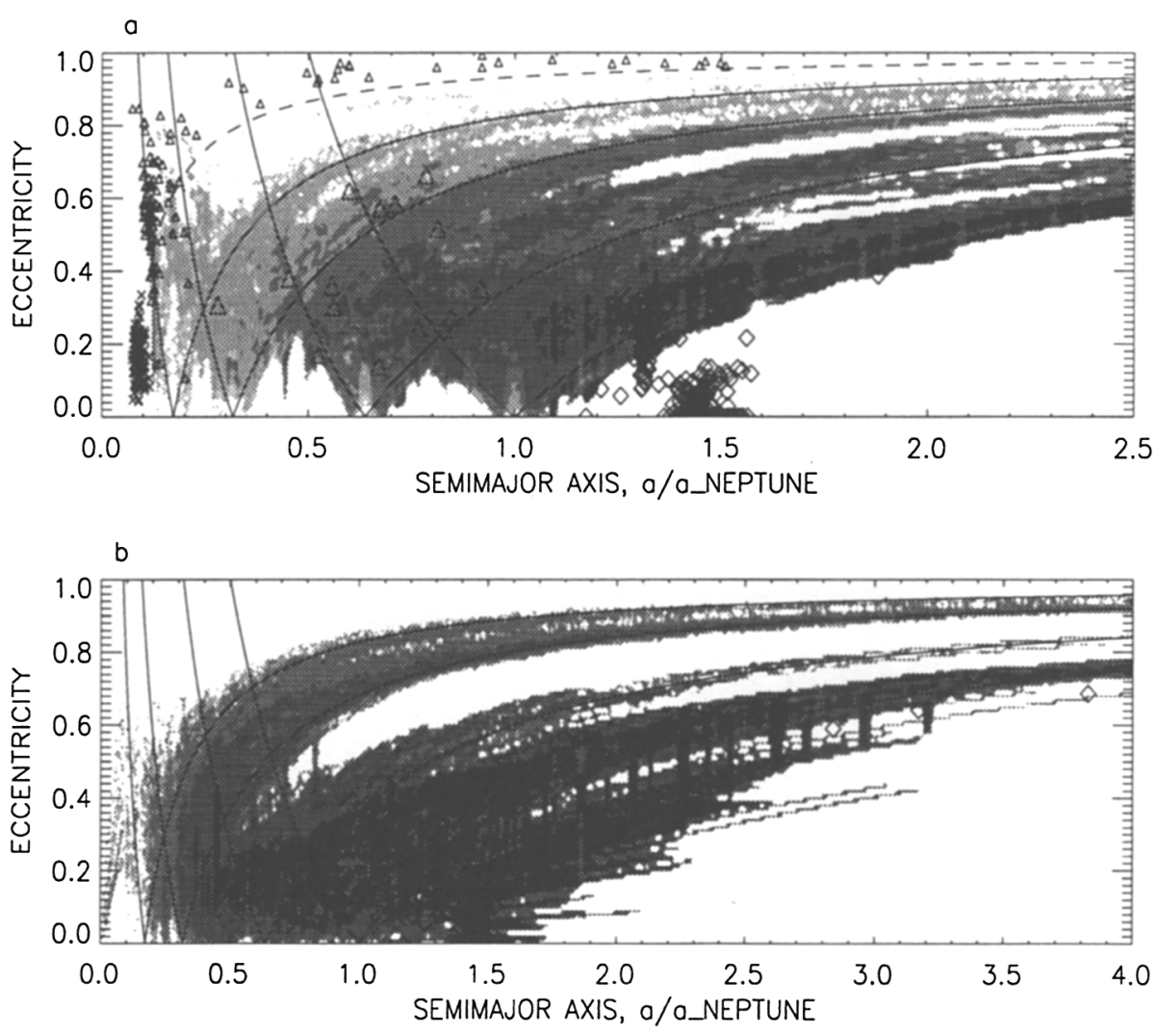

Figure 1. The simulated cometary and dust populations in coordinates 'eccentricity - semimajor axis'. To represent the number of comets/dust particles in each cell, a logarithmic scale with 6 grey levels is used, i.e. each shade differs 10 -fold from the neighboring one. The boundaries of the so called crossing zones (where the orbits of comets and planets are crossed) are shown by heavy lines. Diamonds stand for the (205) Kuiper belt objects.

(a) - 2D density of the simulated cometary population in the outer Solar System (eight-planet approximation) (OGT 2000a). Four cometary belts of the giant planets with resonant structures can be seen. The region occupied by visible comets (perihelion distances $<2 \mathrm{AU}$ ) is located above the dashed line. The known asteroids of the main belt (100 objects) are shown by crosses, small triangles stand for shortperiod comets (112 objects), and large triangles stand for Centaurs (15 objects).

(b) - 2D density of the simulated population of the kuiperoidal dust (eight-planet approximation, 200 sources) (GOMT 2000a). Four cirsumsolar dust belts, with the scattered components along the boundaries of each planet's crossing zone, containing rich resonant structures can be seen near the orbits of the giant planets. 

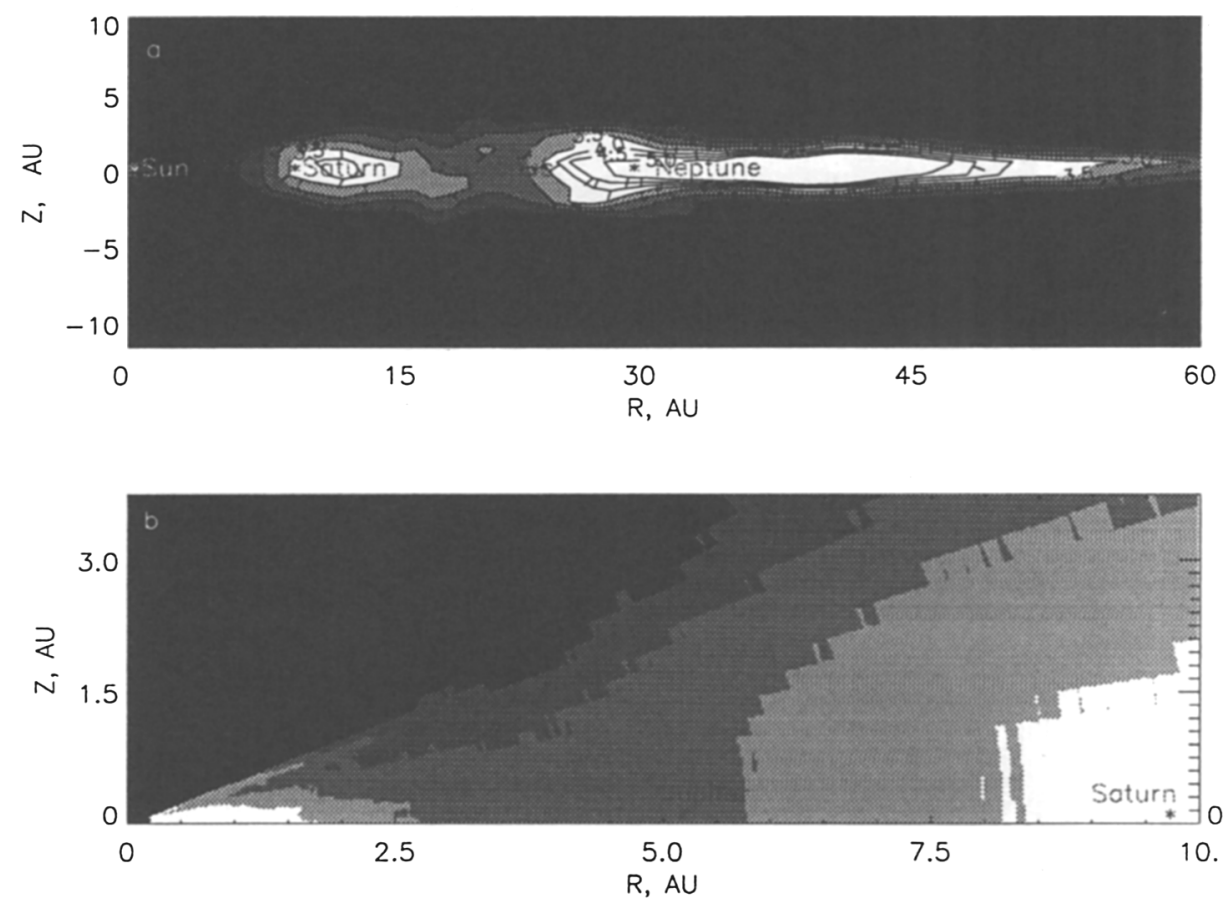

Figure 2. (a) - An edge-on view on the simulated distribution of kuiperoidal dust in the Solar System produced by $133 \mathrm{KBOs}$. This 3D model is constructed on a rectangular $200 \times 200 \times 200$ grid with $8 \cdot 10^{6}$ cells containing $0.5 \cdot 10^{11}$ particle positions.

(b) - The simulated distribution of kuiperoidal dust in the inner part of the Solar System (GOMT 2000a). 200 sources of dust have been used. This 3D-model is based on a spherical $45 \times 180 \times 245$ grid with $2 \cdot 10^{6}$ cells containing $0.6 \cdot 10^{11}$ particle positions.

Using our approach, we have reconstructed the spatial structure of the IPD cloud in the Solar System between 0.5 and 100 AU. Our simulations offer a 3 -D physical model of the kuiperoidal dust cloud based on $(2-8) \times 10^{6}$ cells containing $(0.5-0.6) \times 10^{11}$ positions of dust particles. Here we present the results concerning the distribution of dust particles (of radius 1-2 $\mu \mathrm{m}$ ) produced by $100 \mathrm{KBOs}$ from both the pericenter and apocenter of each. Figs. 2a,b show the spatial structure of kuiperoidal dust up to $60 \mathrm{AU}$. Our simulations reveal a new dust component in the form of gravitationally scattered kuiperoidal dust in the belts near Jupiter and Saturn. This scattered population is basically nonresonant, is highly inclined and possesses large eccentricities. A major part of this component is ejected from the Solar System while passing by Saturn's and Jupiter's orbits.

The other, resonant component of kuiperoidal dust is responsible for the regions of elevated dust density. These resonant dust belts, especially near 
Neptune's orbit, can be seen in Fig. 1b. Our simulations used two particle sizes $(1-2 \mu \mathrm{m}$ and $5-10 \mu \mathrm{m})$. The smaller the particle size, the smaller is the contrast between resonant structure and background, in accordance with Liou \& Zook (1999). There is a remarkable density minimum between Mars and Jupiter. This minimum, which is seen more clearly in Fig. 2b, is due to the fact that Jupiter either ejects from the Solar System or transfers to more inclined and eccentric orbits an appreciable part of the dust drifting toward the Sun. An increase of dust number density in the region between Mars and Earth is explained by the role of the P-R drag, which results in a density profile $\propto R^{-1}$. Finally, as a major result, we find that the number density of kuiperoidal dust increases with heliocentric distance between 4 and $10 \mathrm{AU}$ but forms a plateau between 10 and $50 \mathrm{AU}$.

\subsection{Which of the Dust Components Prevail in the Solar System?}

Our hypothesis that the kuiperoidal dust dominates in the Solar System (certainly, in its outer regions) has been verified by confronting it with available data on the dust distribution in the Solar System, both outer and inner. Two fundamental facts need to be mentioned: (i) at Earth, the dust density profile $R^{-a}$ has the exponent $a=1.3$ (Divine 1993); and (ii) between Jupiter and Neptune, the dust density is almost constant (Humes 1980; Gurnett et al. 1997).

Fig. 3 shows, for various possible components of the IPD cloud, the number density of the simulated dust distribution in the ecliptic plane as a function of heliocentric distance. Within the distance interval of $0.5-1.5 \mathrm{AU}$, an averaged density profile $R^{-a}$ has the exponent $a=1.5-1.7$ for the cometary dust; $a=1.4$ for the kuiperoidal dust, and $a=1.0$ for the asteroidal component. We find that both the two-component and three-component models described above give us the exponent $a$ very close to $a=1.3$ observed at Earth. However, the difference between the two models, only marginal at Earth, becomes very significant at large heliocentric distances. Fig. 3b demonstrates the simulated dust density profile for different components of the IPD cloud at heliocentric distances up to $60 \mathrm{AU}$. Both the cometary dust and small asteroidal dust decrease their number densities in the distance range of $10-30 \mathrm{AU}$ as $R^{-(2.4-3.3)}$, whereas that of the kuiperoidal dust changes insignificantly (as $R^{-0.05}$ ). Thus, our hypothesis that the kuiperoidal dust dominates in the outer Solar System explains the Pioneer and Voyager data fairly well, while the traditional view that the bulk of the IPD cloud is produced by the Jupiter family comets is contradicted by the available data from dust detectors, as is clearly seen in Fig. 3c.

\section{Modeling the Zodiacal Light}

\subsection{Fitting the COBE/DIRBE Data}

We have computed the contribution to the zodiacal light from each of the components of the IPD cloud described in Secs. 3 and 4. Figs. 4a,b show the results from these computations. Also shown is a comparison with some representative DIRBE data on the zodiacal light, where the comparison is constructed by a weighted mixture of the various components. As can be seen from the ratio $I_{\text {ecliptic }} / I_{\text {pole }}$ shown in Fig. $4 \mathrm{a}$, both the asteroidal and kuiperoidal components 

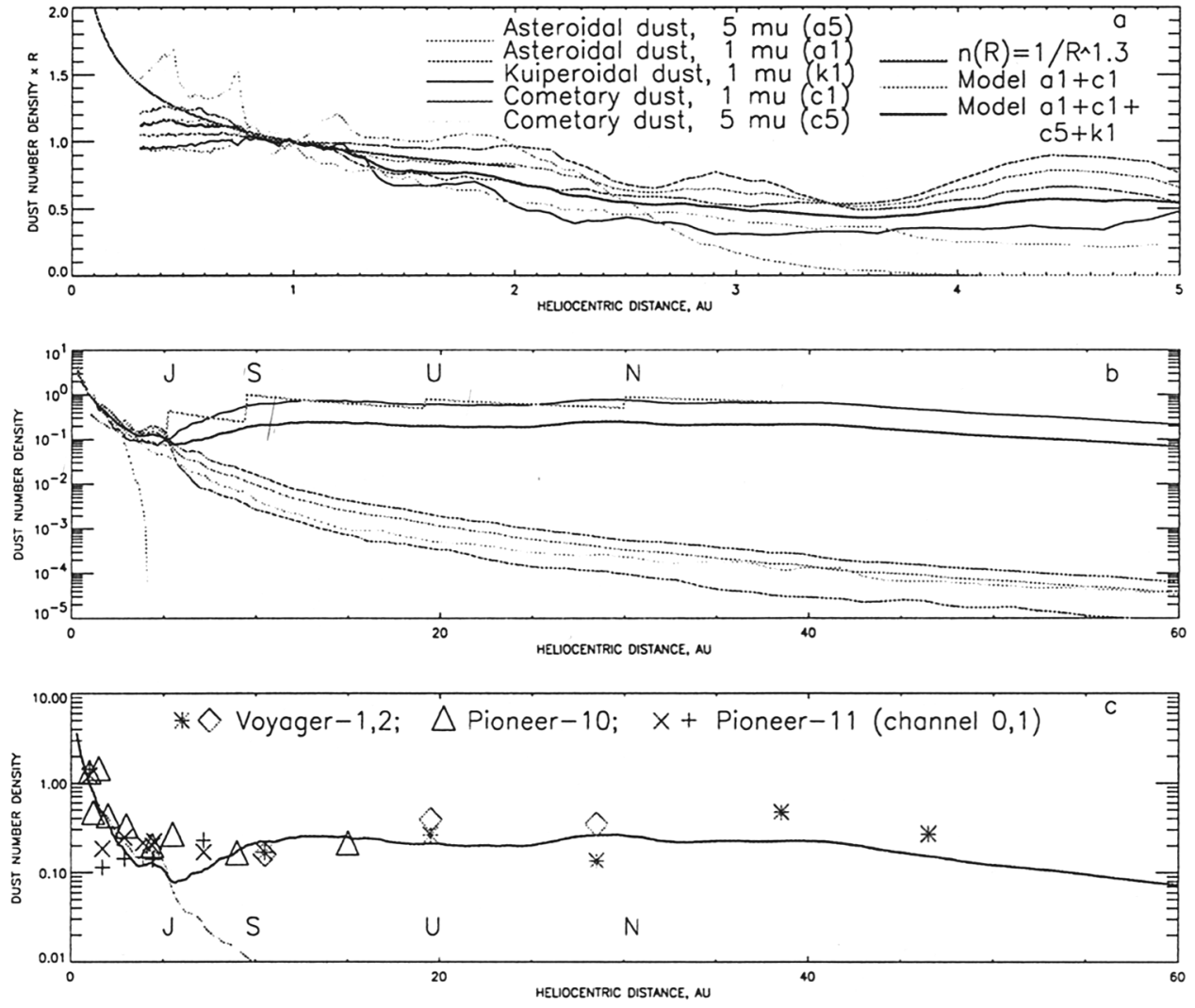

Figure 3. The simulated number density of various components of the IPD cloud in the ecliptic plane (the scale is arbitrary) as a function of heliocentric distance $R$.

(a) $-0.3-5 \mathrm{AU}$. The ordinate is multiplied by $R$ so that the fiducial density run $R^{-1}$ yields a constant. Remarkably, the number density of large asteroidal grains sharply decreases at $R>2 \mathrm{AU}$, in accordance with our analytical model (GOMT 1997a).

(b) $-0.3-60 \mathrm{AU}$ distance range. The number density of kuiperoidal dust remains approximately constant at 10-40 AU. The saw line offers a simple explanation to this fundamental result: the number density of dust drifting under the P-R drag toward the Sun increases as $R^{-1}$, but the planets decrease it by ejecting particles from the Solar System or putting them into more inclined or eccentric orbits. For visualization purposes, the influence of the planets is shown as single jumps, although the actual gravitational scattering is much smoother.

(c) $-0.3-60 \mathrm{AU}$ distance range. Comparison of the simulated dust density confronted with the dust detector data indicates that the model without the kuiperoidal component (grey line) strongly disagrees with the data, whereas the model with the kuiperoidal component (heavy line) is in a fair agreement with them. 
have a flatter shape than the actual IPD cloud. In contrast, the Jupiter family comets create a cloud thicker than the actual one.

As seen in Fig. 4b, a three-component ('asteroidal-cometary-kuiperoidal') physical model of the IPD cloud describes the COBE data with an average accuracy of $0.85 \%$. Although this model employs three free physical parameters (which actually can be determined from observations), it offers an accuracy that is comparable to the best phenomenological model of the zodiacal cloud using about 50 free parameters (Kelsall et al. 1998).

\subsection{Modeling the Zodiacal Light Far from Earth}

Space observations far from Earth, e.g., at $3 \mathrm{AU}$, would offer substantial improvements in the zodiacal light emission and scattering (Mather and Beichman 1996). Unfortunately, a rather accurate multi-parameter model of the zodiacal brightness derived by Kelsall et al. (1998) from the COBE data cannot be reliably extrapolated to heliocentric distances as large as 3 AU. Meanwhile our physical modeling of the zodiacal cloud makes it possible to evaluate quantitatively the zodiacal light emission and scattering throughout the Solar System (GOMT 2000b).

Using the inferred distribution of the zodiacal dust, we have computed a variety of zodiacal light maps, both for thermal emission and scattered components, at different locations $(R, Z)$ of the observer. Fig. 5 is a representative illustration of the brightness of asteroidal dust at $5 \mu \mathrm{m}$ as a function of latitude $\varphi$ and longitude $\lambda$ (in the telescope's frame) given on a logarithmic scale (the neighboring contour intensities differ by $\sqrt{e}$ ); the numerical labels are dimensionless. The Sun's position is $(0,0)$ for $Z=0$ and is shifted to a negative value of $\varphi$ for $Z=0.25 \mathrm{AU}$. At each location, there is a minimum in the zodiacal light which can be seen as a 'dark spot' (or several spots). The positions of those minima are explained by an interplay between dependencies of emissivity upon density and temperature.

\subsection{Warp of the Zodiacal Cloud}

The smooth zodiacal dust cloud is inclined to the ecliptic plane by $2.03 \pm 0.017^{\circ}$ (Kelsall et al. 1998). Since during the annual Earth motion the bulk of the zodiacal dust is positioned either above the ecliptic plane or below it, the zodiacal light turns out to be variable.

The cause of the warp of the zodiacal cloud is as yet a puzzle. Interestingly, a similar warp has been recently discovered in the circumstellar disk around Beta Pictoris (Heap et al. 2000 and references therein). We point out that the observed warp in both cases is caused by the presence of a massive planet such as Jupiter. To prove this, we have plotted the North pole emission using both data from the 41-week helium-cooled period and (so far virtually unused) data of a more extended (about 3 year), 'warm' period of COBE mission (Kelsall et al. 1998). We find a well-pronounced 12-month period, which indicates that an inclined part of the zodiacal cloud is stationary in the inertial frame. Therefore, the warp of the cloud could be associated with the influence of Jupiter on a nonresonant part of the cloud, and the annual variations of the zodiacal emission are induced by the Earth's orbital motion through the warp. 

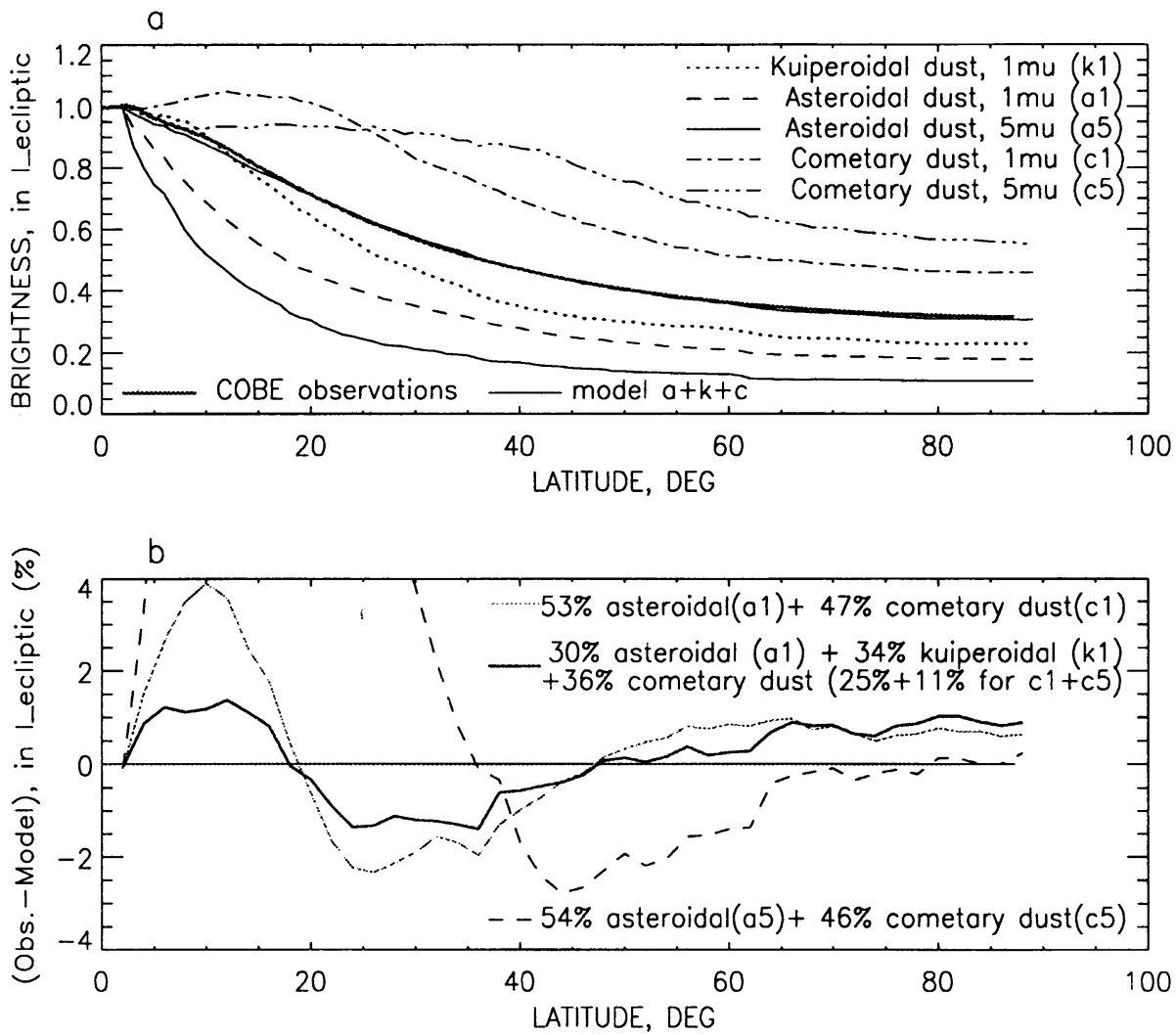

Figure 4. (a)-Infrared emission of different components of the simulated IPD cloud and (b) - a detailed comparison of our models with the COBE/DIRBE data at $\lambda=25 \mu m$, elongation $=90^{\circ}$. The asteroidal dust is assumed to be produced by 220 asteroids, the cometary dust - by 388 comets, and the kuiperoidal dust - by 200 sources. The total number of the computed particle positions is more than $10^{11}$. The cometary particles form a thicker cloud, whereas the asteroidal as well as kuiperoidal particles - a thinner cloud than the observed one. Maximal and average deviations from the COBE data are, respectively, $18 \%$ and $7 \%$ for the "a5 + c5" asteroidal-cometary model and $4 \%$ and $1.5 \%$ for the "a1 + c1" model. All other combinations of (a1, a5, c1, c5) components would only give intermediate values of the deviations. Meanwhile the "asteroidal-cometary-kuiperoidal" model fits the data much better. Disagreement with the data is $0.85 \%$ on average and never exceeds $1.4 \%$. 

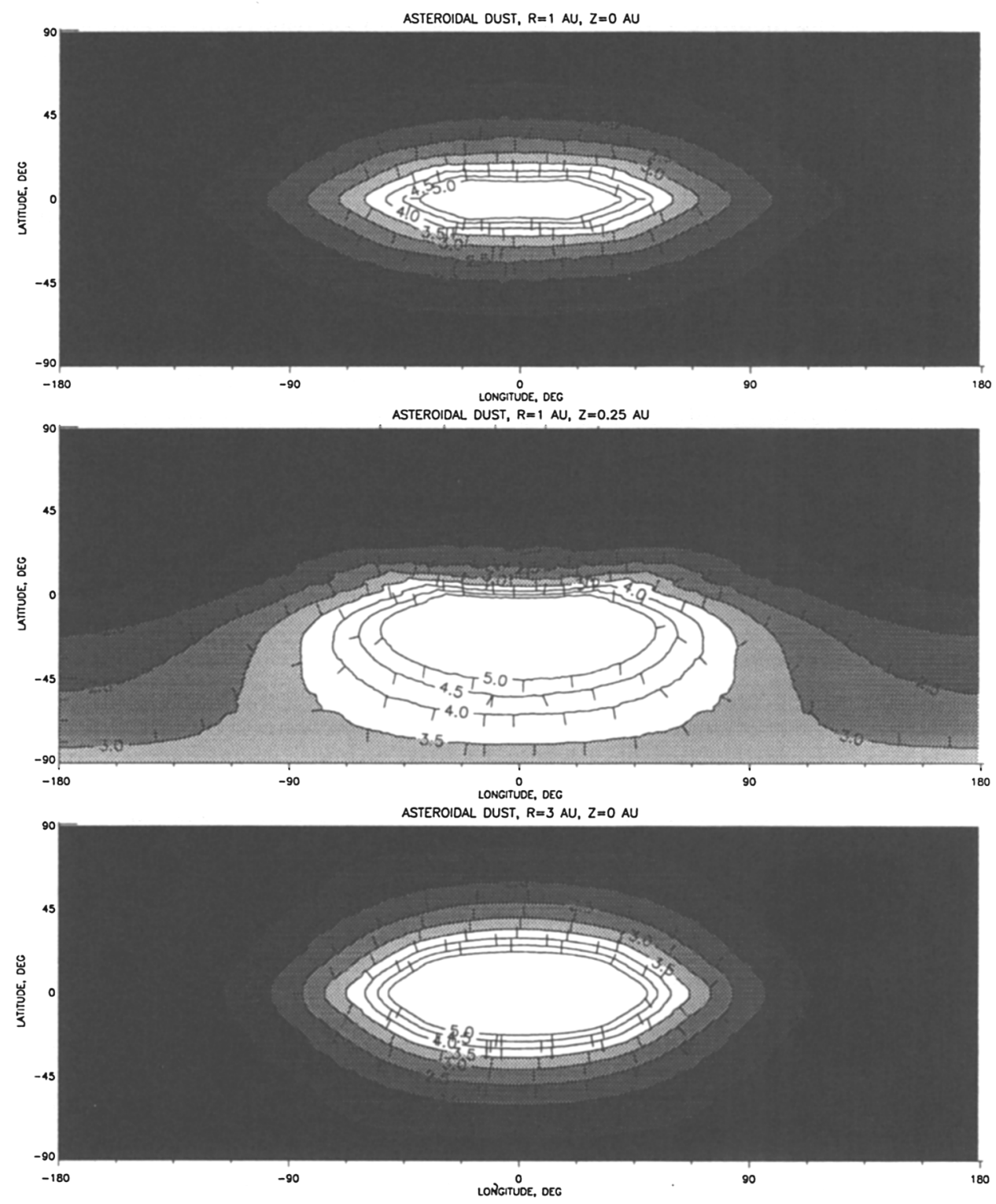

Figure 5. Emission of small $(r=1-2 \mu \mathrm{m})$ asteroidal dust in the $5 \mu \mathrm{m}$ band 
Our numerical simulations confirm the efficiency of this process. Since the warp of the disk around $\beta$ Pic can be visualized more easily than that around the Sun, it would be instructive to address our numerical simulation of the $\beta$ Pic dust disk to illustrate the warp induced by a Jupiter-like planet in an orbit inclined to the disk (see Gorkavyi et al. 2000c). As shown there, a comparison of that modeling with the STIS observations makes the proposed interpretation quite plausible.

\subsection{Simulations of Zodiacal Dust in Circumstellar Disks}

While the warping of a dusty disk, like that surrounding $\beta$ Pic, seen edge-on might serve as a signature of an embedded planet, simulations of circumstellar disks seen face-on need a different approach. The major factors which cause the re-distribution of dust visible in the circumstellar disk in the presence of an embedded planet are: (i) gravitational scattering by the planet, which produces a central cavity (a 'hole') and (ii) resonances, which produce asymmetry in the dust distribution in the form of clumps, arcs, rings, etc. As the dust passes by the planets in its infall, it interacts with them by accumulating in the outer planetary resonances (Liou \& Zook 1999; OGMT 2000). As we demonstrate in OGMT (2000), the resonant structure in the dusty circumstellar disks seen face-on, like Vega or Epsilon Eridani, could serve as an efficient tool of planet detection in those systems.

Thus, along with the ability to compute an improved physical model of the IPD cloud in the Solar System and to explain some key aspects of the available data with them, our tools enable us to predict important details in the dust structure near other stars to be tested in future observations. For instance, using our modeling, we predict that the resonant asymmetric feature revolves around $\epsilon$ Eri and Vega with an angular velocity measurable within a few years (OGMT 2000; Gorkavyi et al. 2000c).

\section{Future Work}

We plan to improve our physical modeling so as to fit the DIRBE data with a precision much better than $1 \%$. To this end, one needs:

- to accurately compute the density distributions of all dust components incorporating particles of 5 to 10 different sizes and accounting for all known as well as simulated sources of dust;

- to account for evaporation and sputtering of dust as a function of heliocentric distance;

- to include the short-term (days to months) variability and small-scale phenomena in the zodiacal cloud.

The last point deserves a more detailed discussion. The time variations found in the zodiacal light are at a level of a few $\%$ for the short $(\lesssim 5 \mu \mathrm{m})$ wavelength bands and of order of 0.5 to $1 \%$ for the longer wavelength bands (Kelsall et al. 1998), i.e., appreciably greater than expected on the basis of known variability in the bolometric output from the Sun. 
The revealed short-term variability might contain contributions of different origins, such as (i) variability of solar wind; (ii) inhomogeneities in the zodiacal cloud along the Earth orbit associated, e.g., with the Earth resonant ring and recent meteoroidal dust or cometary tails; and (iii) dust-source dependence and/or dust response to UV or particle change with time as the solar-wind and/or UV heating 'cook' the particles.

Given that the IPD inhomogeneities are located at distances from Earth not exceeding several AU, their parallaxes are as large as tens of degrees. This provides a unique opportunity to reconstruct, via 3-D 'computer tomography', a 3-D map of small-scale inhomogeneities in the zodiacal cloud using the 41 week data.

Knowledge of the variability and local structure of the IPD cloud would allow us to take the next important step and to determine the absolute value of the zodiacal emission. Indeed, in a given direction on the sky, the Galactic emission is constant, whereas the zodiacal emission varies in time. The physical modeling of the emission amplitude in the above direction could enable us to disentangle the Galactic and zodiacal contributions. As a result, a very accurate fitting of the zodiacal light would be possible, able to solve two major problems: (i) to derive the basic physical parameters of the zodiacal cloud containing valuable information concerning the structure, dynamics, and origin of this cloud, and (ii) the residuals would make it possible to constrain or even evaluate the contribution of the extragalactic infrared background.

\section{Summary and Conclusions}

We have developed a physical model of the zodiacal cloud incorporating the real dust sources of asteroidal, cometary, and kuiperoidal origin.

We have demonstrated the efficiency and power of our codes by performing the following simulations to derive:

(i) a distribution of the scattered comets, which enables us to reveal the four 'cometary belts' associated with the orbits of four giant planets, which are expected to contain 20-30 million cold comets;

(ii) a detailed analysis of a rich resonant structure found in these belts, which predicts the existence of gaps similar to the Kirkwood gaps;

(iii) a 3-D physical model of the IPD cloud, which explains the available data of Pioneer and Voyager dust detectors;

(iv) a zodiacal light distribution in the Solar System, which fits the COBE data with an average accuracy of $0.85 \%$, and

(v) a resonant structure in dusty circumstellar disks of Vega and Epsilon Eridani and a warp in dusty disk of Beta Pictoris considered to be a signature of embedded extrasolar planets.

Under a set of reasonable assumptions, it seems safe to conclude:

1. The kuiperoidal dust plays a role more important than previously recognized. It appears to account for the space dust observations beyond $6 \mathrm{AU}$, while near Earth it could possibly contribute as much as $1 / 3$ of total number density (1/4 of surface density) and $1 / 3$ of the zodiacal emission near the ecliptic.

2. The two other components of the IPD cloud, the cometary and asteroidal dust contribute respectively $36 \%$ and $30 \%$ of the number density and the zodiacal 
emission (at ecliptic) near Earth. The cometary particles contribute $60 \%$ to the surface density of the IPD cloud near Earth. A solely two-component model (i.e. without the kuiperoidal dust) would give a worse fit for the dust distribution at Earth and would fail entirely for the outer Solar System.

3 . Further simulations of resonances associated with the planets embedded in dusty circumstellar disks could enable a breakthrough in the understanding of the circumstellar disk structure and lead to possible planet detection long before direct imaging can find them.

Further development of a multicomponent, high-precision (at the level of a few $\times 0.1 \%$ ) model of the IPD cloud would permit solving, at a new qualitative level, a number of key astronomical problems:

- to get important information about the major physical effects operating in the Solar System, such as the PR drag, resonant captures, gravitational scattering, the role of interstellar particles (including their collisions with the IPD particles), evaporation of dust, efficiency of dust production at different distances from the Sun, etc.;

- to evaluate the parameters of yet undiscovered cometary and asteroidal populations contributing to the origin of the zodiacal cloud;

- to get a reliable template for exo-zodiacal clouds (circumstellar dusty disks) as a basis for revealing embedded exo-planets;

- to help in interpreting and guiding a number of space missions with dust collectors, such as CASSINI and STARDUST;

- to help in planning the targets for space infrared telescopes, such as SIRFT and NGST;

- to improve evaluations of micro-meteoroid impacts for spacecraft;

- to subtract the zodiacal contribution from the COBE DIRBE data with high precision to evaluate/constrain the Cosmic Infrared Background.

Acknowledgments. This work would have been impossible without my collaboration with Nick Gorkavyi, John Mather, and Tanya Taidakova. I am thankful to Thomas Kelsall for many fruitful discussions. Support by NASA Grant NAG7065 to George Mason University is acknowledged.

\section{References}

Backman, D. E., Dasgupta, A., \& Stencel, R. E. 1995, ApJ, 450, L35

Dermott, S. F. et al. 1994, Nature, 369, 719

Dermott, S. F. et al. 1996, in ASP Conf. Ser. Vol. 104, Physics, Chemistry, and Dynamics of Interplanetary Dust, ed. B. Gustafson \& M. Hanner (San Francisco: ASP), 143

Divine, N. 1993, J. Geophys. Res., 98E, 17029

Flynn, G. J. 1994, Lunar \& Planetary Science, XXV, 379 
Flynn, G. J. 1996, in ASP Conf. Ser. Vol. 104, Physics, Chemistry, and Dynamics of Interplanetary Dust, ed. B. Gustafson \& M. Hanner (San Francisco: ASP), 171

Gorkavyi, N. N., Ozernoy, L. M., \& Mather, J. C. (इ GOM) 1996, in ASP Conf. Ser. Vol. 104, Physics, Chemistry, and Dynamics of Interplanetary Dust, ed. B. Gustafson \& M. Hanner (San Francisco: ASP), 43

Gorkavyi, N. N., Ozernoy, L. M., \& Mather, J. C. (三 GOM) 1997, ApJ, 474, 496

Gorkavyi, N. N., Ozernoy, L. M., Mather, J. C., \& Taidakova, T. (三 GOMT) 1997a, ApJ, 488, 268

Gorkavyi, N. N., Ozernoy, L. M., Mather, J. C., \& Taidakova, T. 1997b, BAAS, 29,782

Gorkavyi, N. N., Ozernoy, L. M., Mather, J. C., \& Taidakova, T. 1997c, BAAS, 29,1310

Gorkavyi, N. N. et al. (झ GOMT) 1998a, Earth, Planets and Space, 50, 539

Gorkavyi, N. N., Ozernoy, L. M., Mather, J. C., \& Taidakova, T., 1998b, BAAS, 30,853

Gorkavyi, N. N., Ozernoy, L. M., Mather, J. C., \& Taidakova, T., 1998c, BAAS, 30,1143

Gorkavyi, N. N. et al. ( $($ GOMT) 2000a, astro-ph/0006435; Planetary Space. Sci. (submitted)

Gorkavyi, N. et al. (三 GOMT) 2000b, in ASP Conf. Ser. 207, The NGST Science and Technology Exposition, ed. E. P. Smith \& K. S. Long (San Francisco: ASP), 462

Gorkavyi, N., Ozernoy, L., Mather, J., \& Heap, S. 2000c, in ASP Conf. Ser. (in press), Disks, Planetesimals, and Planets, ed. F. Garzon et al. (San Francisco: ASP); WWW e-print astro-ph/0005347

Gurnett, D. A. et al. 1997, Geophys. Res. Lett., 24, 3125

Haug, U. 1958, Zeitschrift für Astrophysik, 44, 71

Hauser, M. G. et al. 1998, ApJ, 508, 25

Heap, S. et al. 2000, ApJ, (in press)

Humes, D. H. 1980, J. Geophys. Res., 85, 5841

Jackson, A. A., \& Zook, H. A. 1989, Nature, 337, 629

Jewitt, D. 1999, Ann. Rev. Earth. Planet. Sci., 27, 287

Kelsall, T. et al. 1998, ApJ, 508, 44

Kessler, D. J. 1981, Icarus, 48, 39

Leinert, L., Roser, S., \& Buitrago, J. 1983, A\&A, 118, 345

Levison, H. F., \& Duncan M. J. 1997, Icarus, 127, 13

Liou, J.-C., \& Zook, H.A. 1999, AJ, 118, 580

Liou, J.-C., Zook, H. A., \& Dermott, S. F. 1996, in ASP Conf. Ser. Vol. 104, Physics, Chemistry, and Dynamics of Interplanetary Dust, ed. B. Gustafson \& M. Hanner (San Francisco: ASP), 429

Malhotra, R., Duncan, M., \& Levison, H. 1999, in Protostars and Planets IV (in press); astro-ph/9901155 
Mather, J. C., \& Beichman, C. A. 1996, in AIP Conf. Proc. 348, Unveiling the Cosmic Infrared Background, ed. E. Dwek (Woodbury: AIP Press), 271

Ozernoy, L. M., Gorkavyi, N. N., \& Taidakova, T. 2000a, Planetary Space Science, 48,993

Ozernoy, L. M., Gorkavyi, N. N., \& Taidakova, T. (三 OGT) 2000b, MNRAS, submitted; an early version posted in astro-ph/9812479

Ozernoy, L. M. et al. (三 OGMT), 2000c, ApJ, 537, L147

Stern, A. 2000, in ASP Conf. Ser., Highlights of Astronomy, JD 4, ed. A. Lemaitre \& H. Rickman (San Francisco: ASP) (in press)

Taidakova, T. 1997, in ASP Conf. Ser. 125, Astronomical Data Analyses, Software and Systems VI, ed. G. Hunt \& H. E. Payne, (San Francisco: ASP), 174

Taidakova, T., \& Gorkavyi, N. N. 1999, The Dynamics of Small Bodies in the Solar Systems: A Major Key to Solar Systems Studies, ed. B. A. Steves \& B. A. Roy (Kluwer Academic Publishers), 393

Valsecchi, G. B., \& Manara, A. 1997, A\&A, 323, 986 